\title{
ON THE ANALYTIC CONTINUATION OF NEWTON SERIES
}

\author{
V. F. COWLING
}

The object of this note is to prove a theorem on the analytic continuation of Newton's series.

THEOREM. Let $h>0$ and $0<B \leqq \pi$. Let $a(w)$ be regular in the region $|\arg w-h| \leqq B$, and there satisfy $\mid a\left(h+R e^{i \psi} \mid \leqq R^{K} \exp (-L R \sin \psi)\right.$ for some $K$ and some $L, 0<L<2 \pi$, and all large $R$. Then if $f(z)$ $=\sum_{n=0}^{\infty} a(n)(-1)^{n} C_{z-1, n}$ has an abscissa of convergence $\sigma<+\infty$, it is entire.

Proof. We have identically

$$
C_{z-1, n}=\frac{(-1)^{n} \Gamma(z) \Gamma(n+1-z) \sin \pi z}{\pi \Gamma(n+1)} .
$$

Denote respectively $I_{1}, I_{2}$, and $C$ the boundaries of a region $\Gamma\left(h_{1}, R^{\prime}\right)$ bounded by the lines $w=h_{1}+R e^{i B}, w=h_{1}+R e^{-i B}\left(0 \leqq R \leqq R^{\prime}\right.$, $h_{1}>h$ and nonintegral) and the $\operatorname{arc}\left|\operatorname{Arg}\left(w-h_{1}\right)\right| \leqq B$ of a circle $\left|w-h_{1}\right|=R^{\prime}$ of integral radius $R^{\prime}$.

Let $D$ be any (fixed) closed bounded region of the complex plane containing an interval of the positive real axis for which $z=r$ $\geqq \operatorname{Max}[\sigma+\delta, K+\delta], \delta>0$, in its interior.

If now $h_{1}$ is chosen sufficiently large, then the equations $w+1$ $-z=K, K=0,-1,-2, \cdots$ will not have a solution $w$ in $\Gamma\left(h_{1}, R^{\prime}\right)$ for $z$ in $D$. The conditions of the hypothesis are clearly fulfilled in the region $\left|\operatorname{Arg}\left(w-h_{1}\right)\right| \leqq B, h_{1}>h$.

Hence for a fixed $z$ in $D$

$$
\frac{a(w) \Gamma(z) \Gamma(w+1-z) \sin \pi z}{\pi \Gamma(w+1)}
$$

is a regular function of $w$ in $\Gamma\left(h_{1}, R^{\prime}\right)$.

It is then simple to show by means of the calculus of residues that (integration being understood in the positive sense) for $z$ in $D$

$$
\begin{aligned}
& \int_{\Gamma_{\left(h_{1}, R^{\prime}\right)}} \frac{a(w) \Gamma(z) \Gamma(w+1-z) \sin \pi z}{\pi \Gamma(w+1)[\exp (2 \pi i w)-1]} d w \\
&=\sum_{n=\left\{h_{1}\right]+1}^{R^{\prime}+\left[h_{1}\right]} a(n)(-1)^{n} C_{z-1, n} .
\end{aligned}
$$

Presented to the Society, September 2, 1949; received by the editors December 6, 1949. 
Set

$$
G(z, w)=\frac{a(w) \Gamma(z) \Gamma(w+1-z) \sin \pi z}{\pi \Gamma(w+1)[\exp (2 \pi i w)-1]} .
$$

We proceed to show that

$$
\int_{c} G(z, w) d w
$$

converges uniformly to zero as $R^{\prime}$ becomes infinite for $z$ in $D$.

On the $\operatorname{arc} c$

$$
|\exp (2 \pi i w)-1|^{-1}=O(1) .
$$

This follows from the periodic character of the function and the fact that $w$ is bounded away from an integer by our choice of $h_{1}$ and $R^{\prime}$.

From the identity

$$
\frac{1}{\exp (2 \pi i w)-1}=\frac{\exp (-2 \pi i w)}{1-\exp (-2 \pi i w)}
$$

it follows that for $w$ on $c$

$$
|\exp (2 \pi i w)-1|^{-1}=O[\exp (2 \pi R \sin \psi)] \text {. }
$$

This inequality is useful for $-B \leqq \psi<0$.

By a well known result from the theory of the gamma function if we let $z$ denote any complex number independent of $w$ contained in a closed bounded region, then we may write

$$
\Gamma(w) w^{z} / \Gamma(w+z)=1+\epsilon(z, w)
$$

where $\epsilon(z, w)$ tends uniformly to zero as $w$ tends to infinity in such a way as to be bounded away from the negative real axis. In the definition of $w^{z}$ we take that branch of $\log w$ which equals zero when $w=1$.

By application of the hypothesis and the relations (3), (4), and (5) to equation (2), we obtain after some simplification for $w$ on $c$ ( $R^{\prime}$ sufficiently large) and $z=r$ on a closed bounded interval of the positive real axis contained in $D$

$$
|G(z, w)|=O\left[R^{\prime K} \exp \left(-L R^{\prime} \sin \psi-r \log R^{\prime}\right)\right]
$$

$$
\begin{array}{r}
(0 \leqq \psi \leqq B) \\
=O\left[R^{\prime K} \exp \left((2 \pi-L) R^{\prime} \sin \psi-r \log R^{\prime}\right)\right] \\
(-B \leqq \psi \leqq 0) .
\end{array}
$$


Hence

$$
\left|\int_{c} G(z, w) d w\right|=O\left[R^{\prime K+1-r}\right] .
$$

It then follows from (7) that the integral along $c$ converges uniformly to zero as $R^{\prime}$ becomes infinite for $z=r$ on a closed bounded interval of the positive real axis for which $r \geqq K+1+\delta, \delta>0$.

Now let $z=r$ be any point of a closed bounded interval of the positive real axis contained in $D$ for which $z=r \geqq \operatorname{Max}[K+1+\delta, \sigma+\delta]$, $\delta>0$ (such an interval exists by our choice of $D$ ). Then it follows from (1) that for $z=r$ on such an interval we may write

$$
J(z, B)+J(z,-B)=\sum_{n=\left[h_{1}\right]+1}^{\infty} a(n)(-1)^{n} C_{z-1, n}
$$

where

$$
J(z, B)=\int_{I_{1}} G(z, w) d w
$$

and

$$
J(z,-B)=\int_{I_{2}} G(z, w) d w .
$$

The uniform convergence of $J(z, B)$ and $J(z,-B)$ under these conditions follows easily from the relations (6).

We consider now under what more extended conditions $J(z, B)$ and $J(z,-B)$ converge uniformly.

In each case a somewhat lengthy but straightforward calculation reveals that for $z=r e^{i \theta}$ in $D$

(9) $\left|\int^{\infty} G\left(z, h+R e^{i B}\right) d R\right|=O\left[\int^{\infty} R^{K-\cos \theta} \exp (-L R \sin B) d R\right]$

and

$$
\begin{aligned}
\left|\int^{\infty} G\left(z, h+R e^{-i B}\right) d R\right| & \\
= & O\left[\int^{\infty} R^{K-\cos \theta} \exp ((L-2 \pi) R \sin B) d R\right] .
\end{aligned}
$$

The uniform convergence for $z$ contained in $D$ of $J(z, B)$ and $J(z,-B)$ follows easily from an inspection of the right-hand members 
of (9) and (10). Since $G(z, w)$ is continuous in $z$ and $w$ for $w$ on $I_{1}$ or $I_{2}$ and $z$ in $D$ and is a regular function of $z$ in $D$ for a fixed $w$ on $I_{1}$ or $I_{2}$, it results that $J(z, B)$ and $J(z,-B)$ define regular functions in $D$.

Since $D$ contains an interval of the positive real axis for which (8) holds it follows that $J(z, B)+J(z,-B)$ provides the analytic continuation of

$$
f(z)-\sum_{n=1}^{\left[h_{1}\right]} a(n)(-1)^{n} C_{z-1, n}
$$

over the region $D$.

As the region $D$ can be chosen to contain any bounded region of the complex plane in its interior, the theorem follows.

UNIVERSITY OF KENTUCKY

\section{NON-NEGATIVE OBSERVABLES ARE SQUARES}

\section{S. SHERMAN}

For $[\mathrm{S}],{ }^{1}$ whose notation and results we use without further comment, the question of whether or not a non-negative observable is the square of an observable is left open. Our positive solution of this question eliminates one of the hypotheses in a pair of propositions [S,Theorem 4 and Corollary 4.1 ] in that paperand so now we know that without exception a pure state of a subsystem may be extended to a pure state of the whole system of observables and that the spectral value of an observable equals the value of the observable in some pure state of the system. This serves to shrink the gap between $\mathcal{S}$, the real linear space of self-adjoint elements of a $C^{*}$ algebra, and $\mathfrak{A}$, a system of observables. Although every $\mathcal{S}$ is isomorphic to an $\mathfrak{A}$, no examples of an $\mathfrak{A}$ not isomorphic to an $\mathcal{S}$ have appeared in the literature, so the gap may be just an apparent one.

THEOREM. In a system of observables the sum of squares of observables is the square of an observable.

Proof. Let $V=\sum_{\alpha=1}^{n} U_{\alpha}^{2}$. By [S, Corollary 1.1] the closed system $\mathfrak{A}(V)$ generated by $V$ (and $I$ ) is isomorphic algebraically and metrically to $C(X, R)$, the collection of real-valued continuous functions on compact Hausdorff space $X$, where $V \leftrightarrow\left(\phi_{V} \mid X\right) \in C(X, R)$ and $\|V\|$

Presented to the Society, December 29, 1949; received by the editors October 26, 1949.

1 [S] refers to the bibliography at the end of the paper. 